\title{
Bildgebende Diagnostik der allergischen bronchopulmonalen Aspergillose: Vergleich von hochauflösender Computertomographie und Kernspintomographie
}

\author{
D. Franzen ${ }^{1}$ \\ A. Boldt ${ }^{2}$ \\ H. J. Sennekamp 3
}

A Comparison of High Resolution Computerized Tomography and Magnetic

Resonance Imaging in the Evaluation of Allergic Bronchopulmonary Aspergillosis

\section{Zusammenfassung}

Neben serologischen Markern gelten strukturelle Lungenveränderungen als typische Diagnose- und Verlaufsparameter der allergischen bronchopulmonalen Aspergillose (ABPA). Infolge der Problematik invasiver und strahlenintensiver Untersuchungstechniken wie der Bronchographie und Computertomographie wurde der alternative Nutzen einer kernspintomographischen Diagnostik der Lunge bei 5 Patienten mit gesicherter ABPA untersucht. Der direkte Vergleich struktureller Veränderungen der Bronchien und des umgebenden Lungengewebes mittels hochauflösender Computertomographie und Kernspintomographie ließ erkennen, dass die niedrige Ortsauflösung und die Art der Datenakquisition der gegenwärtigen kernspintomographischen Bildgebung nur unzureichend in der Lage sind, Ausmaß und Aktivität der ABPA wiederzugeben. Die MR-Untersuchung kann daher nicht als geeignetes Verfahren zur Diagnose oder Verlaufsbeurteilung der ABPA eingesetzt werden.

\section{Abstract}

Apart from serologic markes structural changes of bronchi and adjacent lung tissue are key factors determining the diagnosis and course of allergic bronchopulmonary aspergillosis (ABPA). Due to problems relating to procedural hazards and radiation exposure using bronchography and computerized tomography we evaluated the efficacy of magnetic resonance imaging of the lung in ABPA. Direct comparison of high resolution computerized tomography (HR-CT) and magnetic resonance imaging (MR) in 5 patients with ABPA revealed that image resolution and data acquisition of present MR technique are insufficient to accurately delineate the extent and activity of structual damage of bronchi and adjacent lung tissue. Although major bronchiectasis may be identified, MR was unable to demonstrate even extensive patchy infiltrates seen in high resolution computerized tomography. At present, magnetic resonance imaging cannot be recommended for determining diagnosis or course of ABPA.
Die allergische bronchopulmonale Aspergillose (ABPA) umfasst ein eher seltenes Krankheitsbild der Bronchien und des umgebenden Lungenparenchyms infolge immunologisch-vermittelter Reaktionen gegenüber Aspergillus fumigatus. Weitaus seltener sind an dem klinischen Syndrom andere Aspergillen-Spezies oder Candidia beteiligt (allergische bronchopulmonale Mykose $=$ ABPM). Die Erkrankung führt zu einer chronisch-entzündlichen Schädigung des Bronchialepithels, die über akute Krankheitsschübe zunächst zur umschriebenen bronchiektatischen Veränderung der proximalen Abschnitte der Bronchien und schließlich zum fibrotischen Umbau des umgebenden Lungenparenchyms führen [1].

Die ABPA stellt unverändert eine besondere diagnostische Herausforderung dar. Obgleich Anamnese, klinisches Bild und serologisch-immunologische Parameter im Vordergrund stehen, ist die radiologische Erfassung struktureller Lungenveränderungen integraler Bestandteil von Erstdiagnostik und Verlaufskontrolle der ABPA. Neben konventionellen Röntgenverfahren erlauben die Bronchographie und die hochauflösende Computertomogra- 
phie (CT) eine subtilere Darstellung und Analyse des Bronchialsystems [2 -4]. Infolge ihrer vergleichsweise größeren Strahlenexposition können sie jedoch nur in der Erstdiagnostik oder bei speziellen Fragestellungen eingesetzt werden. Die vorliegende Untersuchung soll daher prüfen, inwieweit eine kernspintomographische Untersuchung der Lunge geeignet ist, die enormen Vorteile der Schnittbildtechnik bei fehlender Strahlenbelastung in der Diagnostik der ABPA zu nutzen.

\section{Methodik}

Die Diagnose einer allergischen bronchopulmonalen Aspergillose stützte sich auf das Vorliegen einer pulmonalen Grunderkrankung wie Mukoviszidose oder Asthma bronchiale, dem radiologischen Nachweis von (flüchtigen) Lungeninfiltraten, Vorliegen einer Blut- oder Sputumeosinophilie, einer positiven Sofortreaktion auf einen Schimmel- oder Hefepilz im Prick- oder i.c.-Test, den Nachweis deutlich erhöhter Gesamt-Immunglobuline (Ig) der Klasse E (>500 U/l) sowie den Nachweis spezifischer IgEund/oder IgG-Antikörper [1,5]. Der Nachweis von ektatischen Veränderungen der proximalen Bronchien („zentrale Bronchiektasie“) gilt als spezifische und Diagnose-sichernde strukturelle Schädigung der Bronchien [6,7].

Im Rahmen der erforderlichen bildgebenden Diagnostik wurde neben der Computertomographie (CT) eine Kernspintomographie (MR) am selben Tag durchgeführt. Die computertomographischen Untersuchungen erfolgten an einem Mehrzeilen-CT (Volume Zoom, Fa. Siemens, Erlangen). Nach Durchführung einer diagnostischen Thorax-Spiral-CT (Kollimation $4 \times 2,5 \mathrm{~mm}$, Pitch 1,5, Inkrement $8 \mathrm{~mm}$ ) mit Kontrastmittelgabe wurde eine HR-Computertomographie angeschlossen (Schichtdicke $1 \mathrm{~mm}$, Tischvorschub $20 \mathrm{~mm}$ ). Die kernspintomographischen Untersuchungen wurden mit einem 1,5-Tesla-MRT (Sonata, Fa. Siemens) in single slice und Atemanhaltetechnik ohne Kontrastmittel durchgeführt. Es erfolgten $\mathrm{T}_{1}$ - und $\mathrm{T}_{2}$-gewichtete Sequenzen in transversaler,

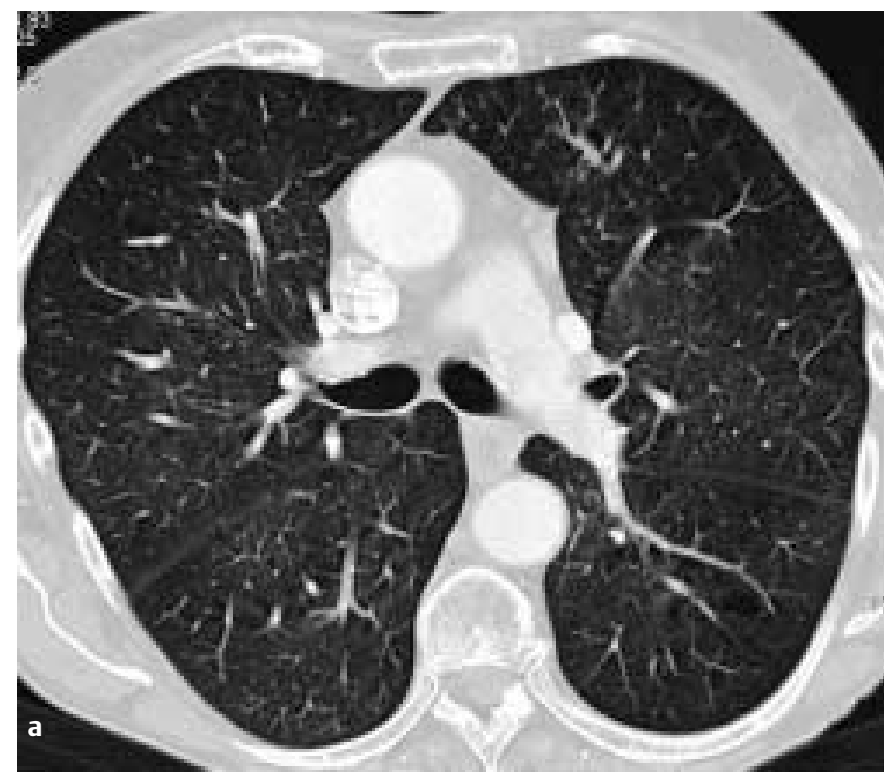

koronarer und sagittaler Schichtführung. Für die Auswertung der Computertomographien wurden sowohl die primären axialen Schichten als auch sekundär rekonstruierte Maximum-IntensityProjektionen (MIP, $10 \mathrm{~mm}$ ) in axialer, koronarer und sagittaler Schichtführung hinzugezogen. Bei den MR-Untersuchungen am Siemens Sonata wurde eine Schichtdicke von $6 \mathrm{~mm}$ gewählt, die sich als Optimum zwischen zu geringer Signal-Intensität und zu großen Partial-Volumen-Effekten erwiesen hatte. Die Darstellung der radiologischen Befunde erfolgte in Anlehnung an die klinische Stadieneinteilung nach Patterson u. Mitarb. [8].

\section{Ergebnisse}

\section{Fallbericht 1}

Bei einer 81-jährigen Patientin fand sich im Rahmen der Abklärung einer langanhaltenden „Bronchitis“ ein ungewöhnlich hohes Gesamt-IgE von 1154 U/l. In der Prick-Testung fand sich neben einer Sensibilisierung gegenüber Hunden, Katzen und Pferden eine deutlich positive Reaktion auf Aspergillus fumigatus. Bei der weiterführenden immunologischen Diagnostik fanden sich spezifische IgE-Antikörper gegen das Epitop Asp 4 EAST Klasse 4 [9]. Lungenfunktionell bestanden eine teilreversible Obstruktion (inspiratorische Vitalkapazität (VC) 2,5 Liter (112\%); Einsekunden-Kapazität $\left(\mathrm{FEV}_{1}\right)$ 0,66 Liter (38\%), nach Akut-Broncholyse 1,0 Liter (58\%); Residualvolumen (RV) 6,4 Liter (294\%); Lungendiffussionskapazität (DCLO) 65\%), die mit der Diagnose eines Asthma bronchiale gut vereinbar war. Im konventionellen Röntgen-Thorax zeigten sich eine peribronchiale Zeichnungsvermehrung beider Lungenunterfelder sowie eine linksseitige dorsobasale Schwielenbildung.

Die nach erfolgreicher Antibiotika-Therapie und bei Beschwerdefreiheit sowie fortbestehend erhöhten IgE-Titern durchgeführte computertomographische und kernspintomographische Untersuchung zeigte keine relevanten pathologischen Veränderungen der Lungenstruktur (Abb.1a u. b). Die im Röntgen-Thorax

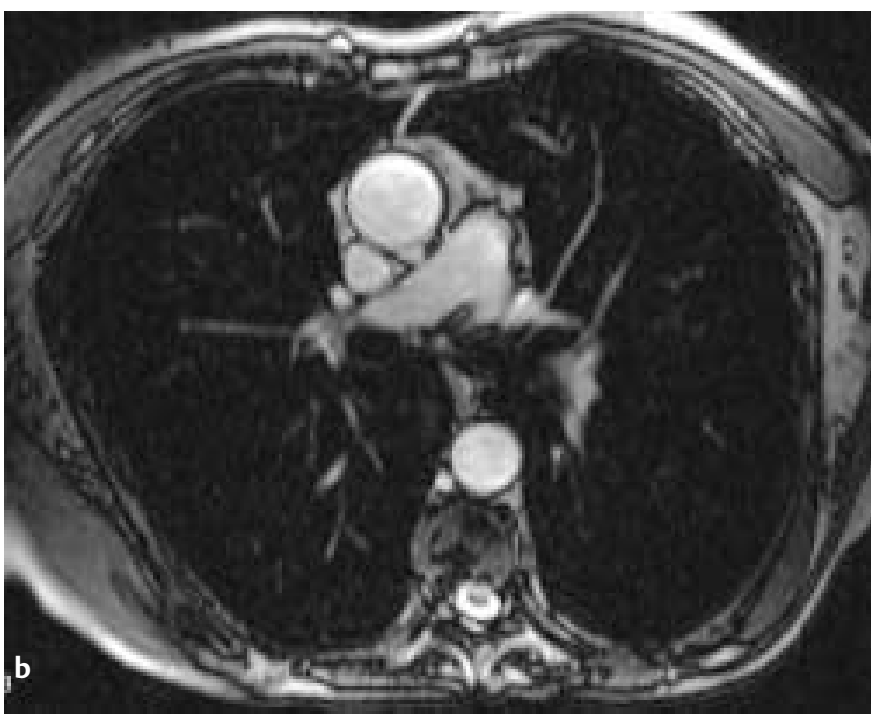

Abb. 1 a HR-CT der Lunge bei seropositiver allergischer-bronchopulmonaler Aspergillose ohne Nachweis der für spätere Stadien typischen zentralen Bronchiektasie. b. korrespondierendes MR-Bild in einer true fisp bright blood Darstellung (6 mm). 
erfasste linksseitige Schwiele kam nur im HR-CT als umschriebene narbige Verdichtung zur Darstellung.

Insgesamt ergab sich die Konstellation einer seropositiven ABPA $[9,10]$. Bei fehlenden klinischen und serologischen Zeichen eines Erkrankungsschubes zeigten sich keine relevanten strukturellen Lungenveränderungen.

\section{Fallbericht 2}

Im Rahmen der Abklärung und Behandlung einer Pneumonie mit Hämoptoe im Dezember 2000 wurde bei einer 45-jährigen Patientin mit lungenfunktionell geringgradig ausgeprägter chronisch obstruktiver Bronchitis ein deutlich erhöhter IgE-Titer auf 1296 U/l gemessen. Bei bekannter Sensibilisierung gegenüber Aspergillen wurden spezifische IgE- und IgG-Antikörper gegen Aspergillus fumigatus festgestellt, im Blutbild fand sich eine Eosinophilie von 15\%. Ein Schub der ABPA im Juni 2001 wurde erfolgreich mit $50 \mathrm{mg}$ Solu-Decortin über 14 Tage behandelt. Im März 2002 kam es zu einem Infekt, der zu einer erneuten radiologischen Diagnostik führte.

Im CT fanden sich eine proximal betonte Bronchiektasie des linken Ober- und Unterlappens sowie geringgradige emphysematische Lungenveränderungen, jedoch keine Hinweise für frische infiltrative oder reaktive Veränderungen. Insbesondere zeigten sich keine milchglasartigen Trübungen der Lungenstruktur. Demgegenüber wurden kernspintomographisch weder die Bronchiektasen noch andere pathologische Lungenstrukturen identifiziert. Auch die im CT fassbare Schwiele der Lingula kam nicht zur Darstellung.

Insgesamt ist bei typischer Konstellation und Diagnose einer allergischen bronchopulmonalen Aspergillose im vorliegenden Fall die typische zentrale Bronchiektasie im CT, nicht jedoch im MR nachweisbar (Abb. 2a u.b). Eine infiltrative Lungenstrukturver- änderung wurde bei beiden Untersuchungen nicht nachgewiesen. Zum Zeitpunkt der Untersuchung handelte es sich um ein Stadium II (Remission) nach Patterson [8].

\section{Fallbericht 3}

Bei einem Marathonläufer wurde im Alter von 33 Jahren erstmals die Diagnose einer Mukoviszidose mit allergischer bronchopulmonaler Candidose im Rahmen der Diagnostik rezidivierender pulmonaler Infekte mit Staphylococcus aureus gestellt. Bodyphlethysmographisch fanden sich eine inspiratorische Vitalkapazität von 2,9 Litern (51\%) und eine totale Lungenkapazität von 8,0 Litern (102\%). Das Residualvolumen betrug 5,1 Liter (258\%). Die 1-Sekunden-Kapazität war mit 1,2 Litern deutlich vermindert (27\%) und sprach nicht auf Akut-Broncholyse mit Salbutamol an (1,1 Liter).

Eine Ende Januar 2001 wegen einer deutlich vermehrten Sputumproduktion mit Nachweis von Staphylococcus aureus durchgeführte Röntgen-Thorax-Untersuchung zeigte neu aufgetretene bilaterale großflächige Infiltrate in beiden Lungenmittel- und Unterfeldern, die klinisch auf eine Antibiotikatherapie rasch ansprach. Zusätzlich wurde im Röntgenthorax der Verdacht auf eine zentrale Bronchiektasie beidseits perihilär und in den Unterlappen sowie einen Lokal-Pneumothorax im linken Lungenoberlappen geäußert. Zum Zeitpunkt der erneuten bildgebenden Diagnostik im März 2001 bestand Beschwerdearmut mit nur geringem Auswurf. Die Blutsenkungsgeschwindigkeit (BSG) war mit $8 \mathrm{~mm} / 1$ h normal. Bei nur mäßig erhöhtem Gesamt-Immunglobulin E von $422 \mathrm{IU} / \mathrm{ml}$ waren die IgG-Antikörper gegen Candida albicans stark positiv, IgA-Antikörper schwach positiv. Im hochauflösenden Mehrzeilen-CT mit Kontrastmittel fanden sich zentrale Bronchiektasien in beiden Oberlappen sowie im Segment 4 links sowie geringer im Segment 8 und 9 rechts. Zusätzlich bestand eine milchglasartige Trübung in den Segmenten 8 und 9 rechts (Abb. 3a). Perihiläre Infiltrate oder ein Pneumotho-
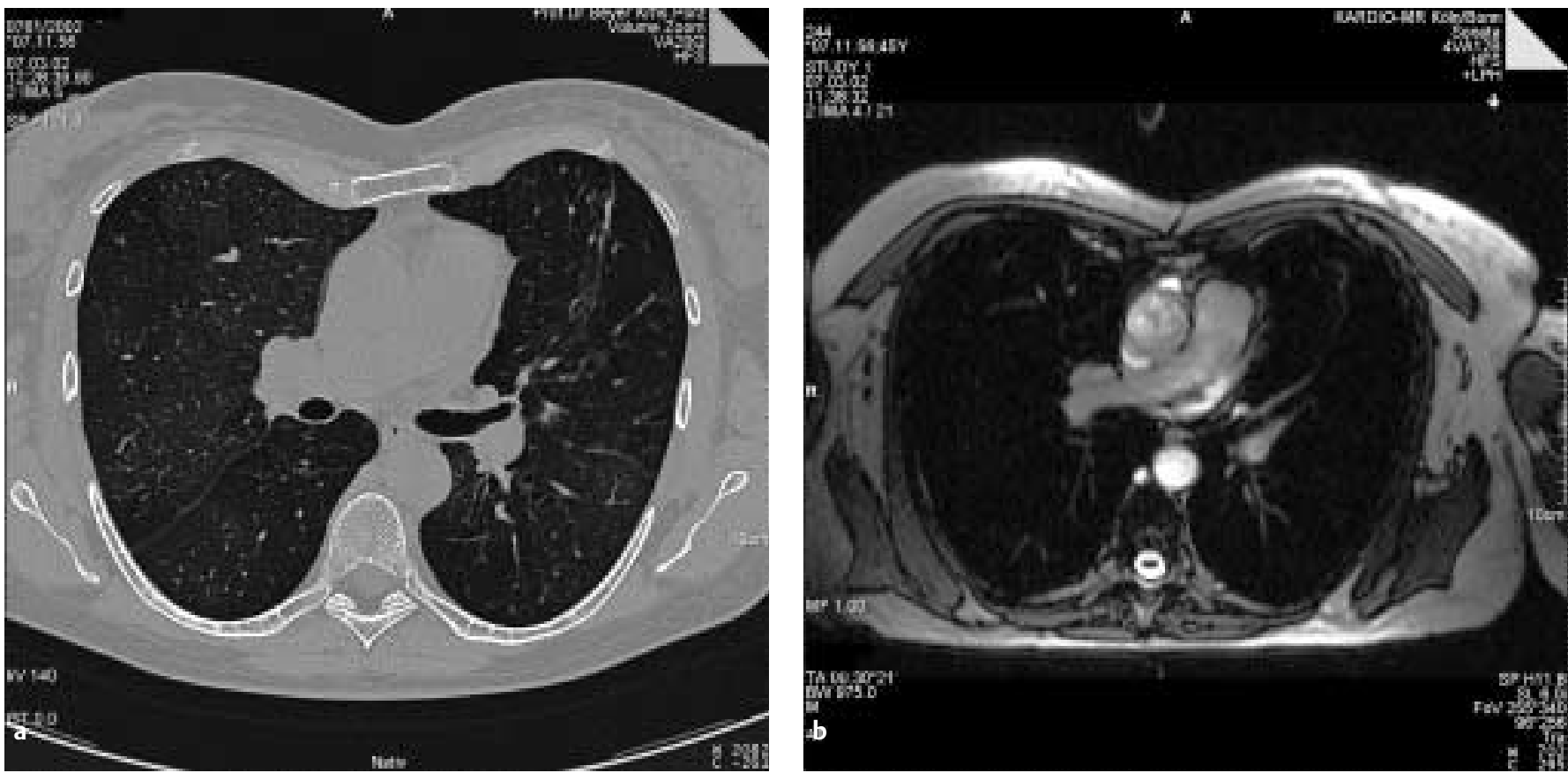

Abb. 2 a HR-CT der Lunge mit mäßig ausgeprägter Bronchiektasie des linken Lungenoberlappens sowie einem geringgradigen Lungenemphysem. b. Im korrespondierenden MR-Bild können keine Bronchiektasen identifiziert werden (true fisp bright blood Darstellung 6 mm). 

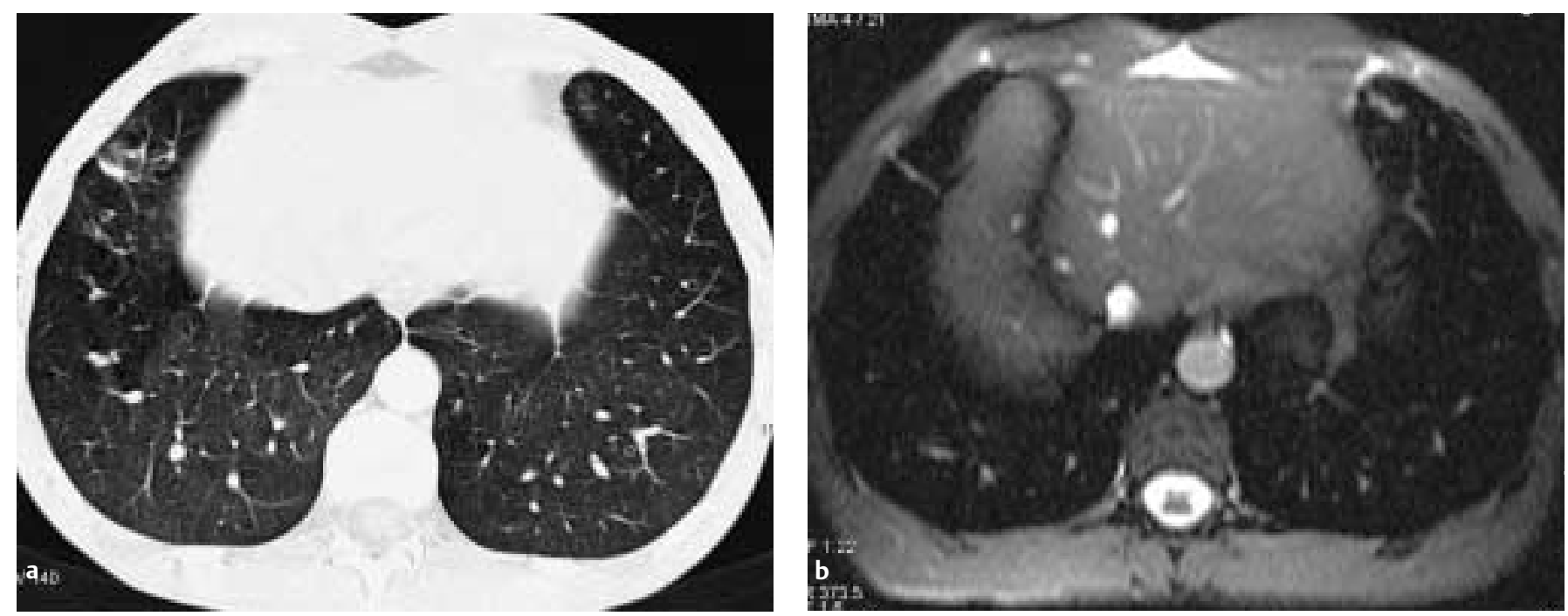

Abb. 3 a Darstellung milchglasartiger Infiltration des Lungenparenchyms im rechten Lungenunterlappen mittels HR-CT. b. Im MR (true fisp 6 $\mathrm{mm}$ ) kommen die Infiltrate nicht zur Darstellung.

rax bestanden nicht. Emphysematöse Veränderungen beider Lungen waren gering ausgeprägt. Am rechten Hilus fand sich ein auf 1,5 cm vergrößerter Lymphknoten. Die Kernspintomographie des Thorax ergab geringgradige Hinweise auf eine, auch hier in den zentralen Abschnitten nachweisbare Bronchiektasie. Die milchglasartigen Infiltrationen wurden mit MR jedoch nicht erfasst (Abb. 3b). Es zeigten sich lediglich verdickte Bronchialwände im Segment 5 links im Bereich der Trachealbifurkation. Pathologische Lymphknoten wurden nicht erfasst.

Ingesamt fanden sich bei klinisch und immunologisch gering aktiver ABPM (Stadium III nach Patterson) eindeutige Aktivitätszeichen im CT der Lunge (milchglasartige Trübung), jedoch keine entsprechenden Veränderungen in der Kernspintomographie. Die computertomographisch fassbaren Bronchiektasien wurden kernspintomographisch in verminderter Intensität, der gering vergrößerte Lymphknoten wurde nicht erfasst.

\section{Fallbericht 4}

Im Rahmen einer Wehrdienst-Untersuchung 1999 wurde erstmals der Verdacht auf eine Lungenerkrankung, im weiteren die Diagnose einer Mukoviszidose mit schwerer exokriner Pankreasinsuffizienz bei einem 18-jährigen Patienten gestellt. Die inspiratorische Vitalkapazität lag bei 3,0 Liter (54\%) und die FEV 1 bei 1,1 Litern (23\%) ohne Ansprechen auf Akut-Broncholyse. Das Residualvolumen war auf 584\% und die totale Lungenkapazität auf $163 \%$ der Norm erhöht, die Diffusionskapazität auf $41 \%$ vermindert. Bei persistierender BSG-Erhöhung ( $52 \mathrm{~mm} / 1 \mathrm{~h}$ ), Nachweis von flächenhaften Infiltraten im Röntgenthorax und im ThoraxCT mit unverändertem Nachweis von Staphylococcus aureus trotz umfangreicher und wiederholter Antibiotika-Therapie, deutlich erhöhtem Gesamt-IgE (2896 U/1) und Nachweis einer Aspergillus-fumigatus-Allergie im Prick und RAST wurde schließlich die Diagnose einer allergischen bronchopulmonalen Aspergillose gestellt und eine orale Steroidmedikation mit $40 \mathrm{mg}$ Decortin/die eingeleitet. Die 2 Monate später durchgeführte Diagnostik ergab eine deutlich rückläufige BSG (17 mm/1 h), eine Auflösung der vormaligen milchglasartigen Infiltrate im Röntgenthorax sowie eine mehrfach dokumentierte
Eradikation des vormals chronischen Befalls mit Staphylococcus aureus.

Wegen rezidivierender deutlicher Erhöhung der BSG wurden im Jahr 2001 mehrfach konventionelle Röntgen-Thorax-Untersuchungen durchgeführt. Diese zeigten durchgehend ein diffuses bilaterales Netzmuster des Lungenparenchyms mit teils streifigen, teils knotigen Verdichtungen von kranial nach kaudal an Intensität abnehmend. Rechts parakardial ließen sich zylindrische und zystische Strukturverschattungen mit Schienengleis-Phänomenen („tramline shadows“) nachweisen. Frische Infiltrate waren zu keinem Zeitpunkt nachweisbar.

Im Rahmen einer klinisch stabilen Phase (IgE $800 \mathrm{U} / \mathrm{l}$, verschwundene Immunoblotlinien gegen spezifisches IgE und IgG gegen Aspergillus fumigatus) wurden im Januar 2002 eine CTund MR-Untersuchung der Lunge durchgeführt. Neben ausgedehnten Bronchialwandverdickungen fanden sich bronchiektatische Bezirke in allen Lungenabschnitten, vorzugsweise in den zentralen Abschnitten beider Oberlappen. In der Kernspintomographie konnten Bronchiektasie und Bronchialwandverdickungen in etwa gleicher Form, im Vergleich zur CT jedoch geringerer Intensität nachgewiesen werden (Abb. $\mathbf{4 a - c}$ ). Wie die transversalen Scans zeigen, ist die Feindarstellung der Bronchiektasie im MR nicht in gleicher Weise möglich wie im CT (Abb. 5a u. b). Die im CT erfassten bullösen und emphysematösen Veränderungen waren im MR nicht nachweisbar (kein Bildmaterial präsentiert).

Im Juli 2002 kam es während eines Kuraufenthaltes auf Amrum zu einem erneuten Schub der allergischen bronchopulmonalen Aspergillose, die infolge symptomatischer Hypoxämie eine nasale Sauerstofftherapie erforderte. Laut Befundbericht der dortigen Klinik wurden mittels konventionellem Röntgenthorax eine Pneumonie und ein Pneumothorax ausgeschlossen. Die erst nach Rückkehr und unter Decortin-Medikation durchgeführte Diagnostik ergab serologisch ein gesamt IgE von $1200 \mathrm{U} / \mathrm{l}$, sowie eine Vermehrung und eine Zunahme der Immunoblotlinien von spezifischem IgG und IgE gegen Aspergillus fumigatus im Sinne eines noch aktiven Schubes. In der Computertomographie der 


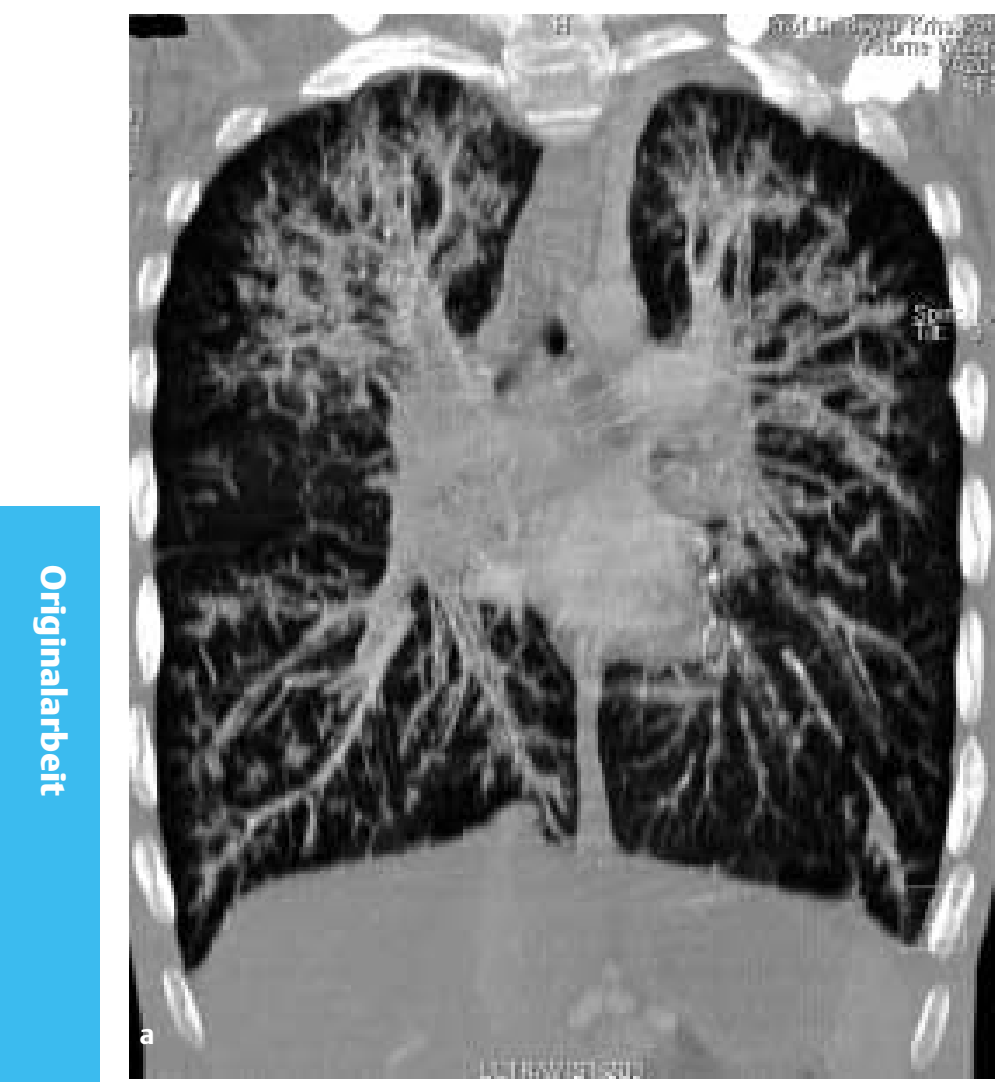

Abb 4 a Im HR-CT (MIP 10 mm) kommen ausgeprägte Bronchiektasien in allen Lungenabschnitten zur Darstellung. Die Bronchiektasien kommen sowohl in einer $\mathrm{T}_{1}$-gewichteten Sequenz (b Turbospin Echo $\mathrm{T}_{1}$ dark blood) wie auch in einer $\mathrm{T}_{2}$-gewichteten MR Bildsequenz (c Turbospin Echo $\mathrm{T}_{2}$ dark blood) zur Darstellung.
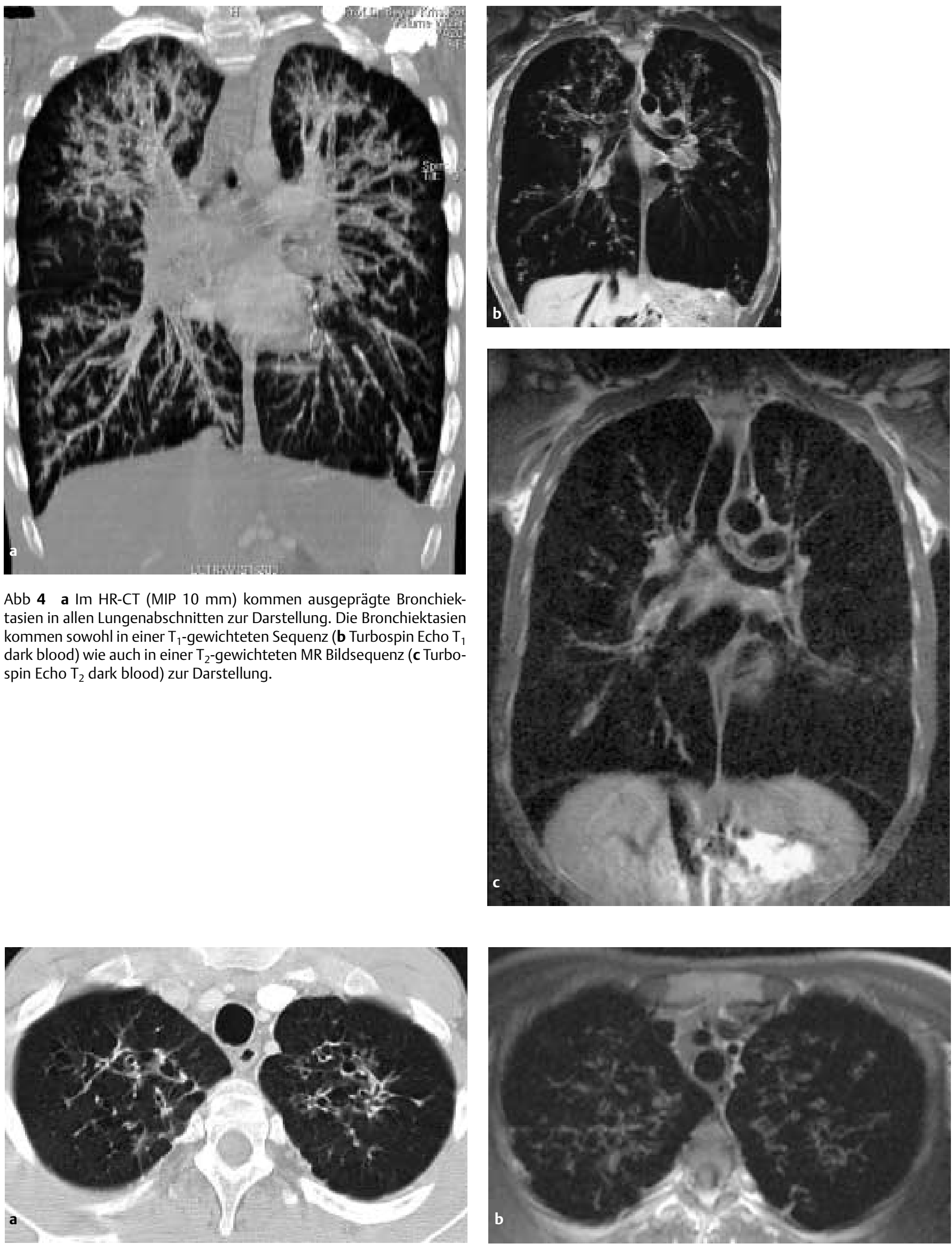

Abb 5 a und b Im HR-CT und im MR (Turbospin Echo $T_{2}$ dark blood) gute Darstellung der zentralen Bronchiektasie bei transversalen Schnitten. 

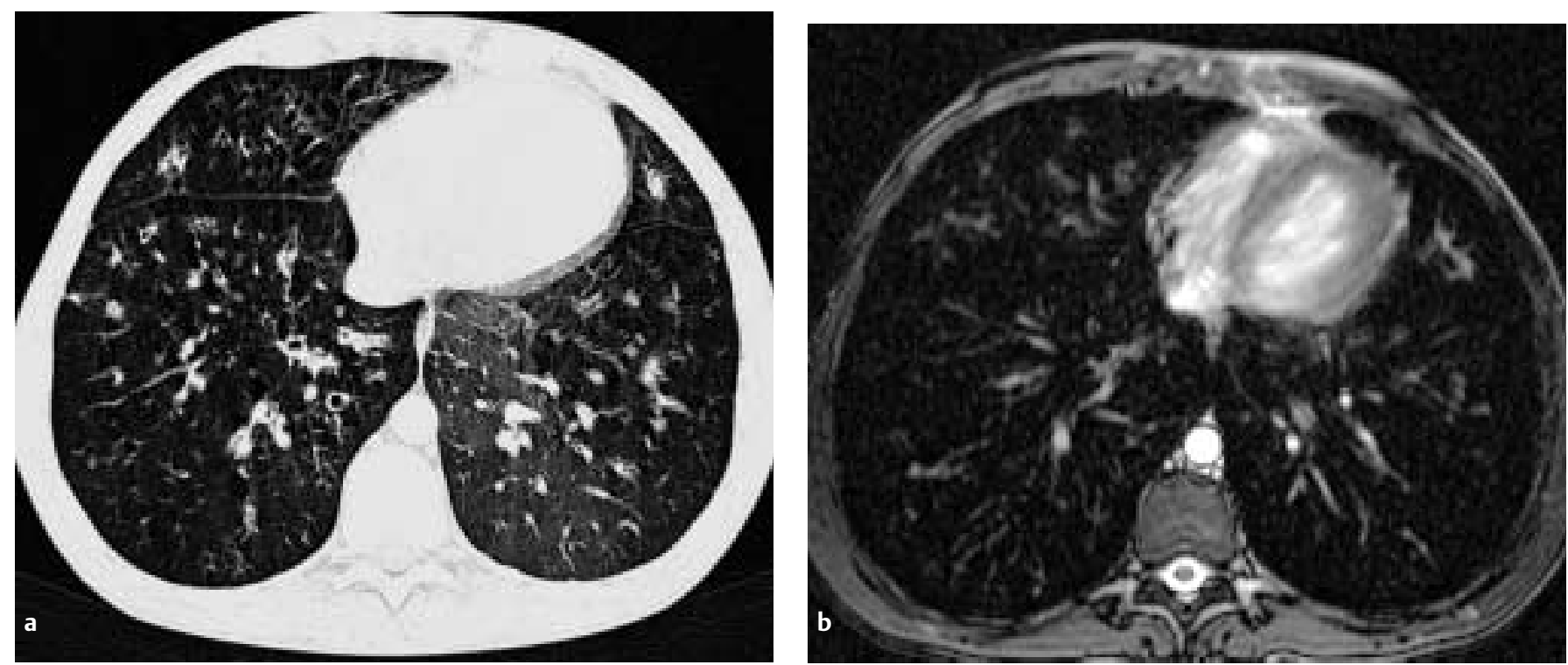

Abb 6 a u. b Im HR-CT Darstellung (a) besonders ausgedehnter milchglasartige Infiltrationen beider Lungenlappen, die im MR (true fisp 6 mm) nicht abgebildet werden (b).

Lunge im August 2002 zeigten sich neben den vorbekannten Veränderungen jetzt ausgedehnte milchglasartige Trübungen des linken Lungenunterlappens sowie von Teilen des Mittellappens und zentraler Anteile des rechten Unterlappens (Abb. 6a). In der Kernspintomographie konnten Bronchiektasie und Bronchialwandverdickungen in vorbekannter Ausdehnung unverändert nachgewiesen werden, die jetzt im CT gut sichtbaren, flächenhaften Infiltraten kamen jedoch kernspintomographisch nicht zur Darstellung (Abb. 6b).

Insgesamt belegen die Verlaufsuntersuchungen im Stadium IV nach Patterson, dass die ausgeprägten strukturellen Veränderungen der Bronchien durch Computertomographie und durch Kernspintomographie gleichermaßen erfasst werden. Die für den Schub der ABPA (Stadium III) typischen milchglasartigen Veränderungen kamen trotz großer Ausdehnung nur in der Computertomographie zur Darstellung.

\section{Diskussion}

Die allergische bronchopulmonale Aspergillose (ABPA) umfasst ein eher seltenes Krankheitsbild der Bronchien und des umgebenden Lungenparenchyms infolge immunologisch-vermittelter Reaktionen gegenüber Aspergillen fumigatus, selten sind auch andere Pilzspezies an dem Syndrom beteiligt. Die Erkrankung führt zu einer chronisch-entzündlichen Schädigung des Bronchialepithels, die über akute Krankheitsschübe zunächst zu umschriebenen bronchiektatischen Veränderungen der proximalen Abschnitte der Bronchien und schließlich zum fibrotischen Umbau des angrenzenden Lungenparenchyms führt [1].

Neben klinischen und serologischen Parametern gelten bildgebende Verfahren als integraler Bestandteil der Diagnostik einer allergischen bronchopulmonalen Mykose. Bis auf ein Frühstadium, der so genannten seropositiven ABPA, finden sich bei allen Patienten Bronchiektasien, die typischerweise die proximalen Segmente der Bronchien vorwiegend in den Lungenoberlappen betreffen und daher als pathognomonisch gelten [3,10-12] (Abb. 4a-c, 5a u. b). Neben diesen chronischen Veränderungen sind die gelegentlich auch protrahiert verlaufenden Krankheitsschübe durch flächenhafte Infiltration des umgebenden Lungenparenchyms charakterisiert. Die computertomographisch milchglasartig imponierenden Trübungen in verschiedenen Lungenabschnitten können ohne Kenntnis der pathophysiologischen $\mathrm{Zu}$ sammenhänge nicht von einer Bronchopneumonie unterschieden werden (Abb. 3a u. 6a).

Neben serologisch-immunologischen Parametern sind serielle Kontrollen mit bildgebenden Verfahren in teilweise kurzen Abständen oder im Schub der ABPA erforderlich, um Diagnose und Therapieerfolg überprüfen zu können. Obwohl die Computertomographie eine weitaus subtilere Diagnostik pathologischer Lungenstrukturen erlaubt, ist deren Einsatz infolge vergleichsweise höherer Strahlenexposition nur bei besonderen Fragestellungen bei den in der Regel jungen Patienten gerechtfertigt. Inwieweit der Einsatz der Niedrigdosis-CT, bei der die Strahlenexposition nur minimal über der Dosis der konventionellen Thoraxdiagnostik liegt [13], hier eine deutliche Erweiterung der Indikation zur CT mit sich bringt, bleibt abzuwarten. Die hier dargestellte Gegenüberstellung von hochauflösendem CT und MR diente daher der Frage, inwieweit die Kernspintomographie der Lunge in der Lage ist, die ABPA-typischen Veränderungen zu erfassen. Im Vergleich zum gewählten Standard, der HR-Computertomographie, kamen bronchiektatische Veränderungen zumindest bei fortgeschrittenem Ausmaß auch kernspintomographisch gut zur Darstellung. Dennoch erschien das örtliche Auflösungsvermögen der MR-Untersuchungen auch bei verschiedenen bildgebenden Sequenzen eindeutig begrenzt (Abb.4a-c). Eine Darstellung infiltrativer Veränderungen des Lungenparenchyms gelang jedoch bei keiner MR-Untersuchung. So wurden weder die milchglasartigen Trübungen, wie sie für einen Erkrankungsschub typisch sind, noch fibrotische Veränderungen bzw. Schwielen oder Lymphknoten ausreichend erfasst. 
Zur Darstellung der Lungenveränderungen wurden MR-Sequenzen und Relaxationszeiten gewählt, für die bereits aussagekräftige Untersuchungen vorliegen [14-16]. Die auch in diesen Studien berichtete Fehlerquote bei der Detektion von Lungeninfiltraten wie auch die bekannten Schwierigkeiten der Abbildung von chronisch-fibrotischen Veränderungen mittels MR decken sich mit den getroffenen Feststellungen bei den hier vorgestellten Kasuistiken.

Seit klinischer Einführung in den 80er-Jahren haben MR-Untersuchungen eine etablierte und ständig wachsende Rolle in der bildgebenden Diagnostik beinahe aller Organe erlangt und in manchen Fragestellungen die konventionelle Computertomographie als primäre Diagnostik verdrängt [17]. In der MR-Diagnostik der Lunge liegen besondere anatomische (viele Luft-Gewebe-Grenzflächen), physikalische (geringe Protonendichte), und physiologische (Diffusions-, Perfusions- und Bewegungsassoziierte Phasenverschiebungen) Faktoren vor, die eine gegenüber dem CT vergleichsweise niedrige Bildqualität bedingen [15]. Neben dem unzureichenden Auflösungsvermögen der derzeitigen MR-Bildgebung sind auch relevante Unterschiede der Datenakquisition bei CT und MR zu berücksichtigen. Während bei der Computertomographie die komplette Lunge 3-dimensional als Datenblock erfasst wird, beruht die hier durchgeführte kernspintomographische Untersuchung auf der Darstellung einzelner, vorher ausgewählter Schichten. Sofern keine Schicht-fürSchicht-Bildgebung durchgeführt wird, können daher kleinere bronchiektatische Veränderung der MR-Darstellung entgehen.

Die hier durchgeführten Untersuchungen zeigen, dass die Kernspintomographie derzeit nicht in der Lage ist, das Spektrum der allergischen bronchopulmonalen Aspergillosen ausreichend darzustellen. Das MR ist daher für die Routinediagnostik und Verlaufskontrolle der ABPA weitgehend ungeeignet und kann derzeit nur bei speziellen Fragestellungen zur Anwendung kommen.
Literatur

${ }^{1}$ Menz G, Willer G, Crameri R. Die allergische bronchopulmonale Aspergillose (ABPA). Pneumologie 2000; 54: 375-384

${ }^{2}$ Grenier P, Frantz M, Musset D et al. Bronchiectasis: assessment by thin-section CT. Radiology 1986; 161: 95 -96

${ }^{3}$ Currie DC, Goldman JM, Cole PJ et al. Comparison of narrow section computed tomography and plain chest radiography in chronic allergic bronchopulmonary aspergillosis. Clinical Radiology 1987; 38: 593-596

${ }^{4}$ Naidich DP, Zinn WL, Ettenger NA et al. Basilar segmental bronchi: thin section CT evaluation. Radiology 1988; 161: 95-96

${ }^{5}$ Sennekamp HJ, Bergmann KC. Allergische Mykosen der Lunge und der Nasennebenhöhlen. In: Fuchs und Schulz (Hrsg.). Manuale allergologicum V 10. 1998: 1-31

${ }^{6}$ Mintzner RA, Rogers LF, Kruglik GD et al. The spectrum of radiologic findings in allergic bronchopulmonary aspergillosis. Radiology 1978; 127: $301-307$

${ }^{7}$ Fisher MR, Mendelson EB, Mintzer RA et al. Use of linear tomography to confirm the diagnosis of allergic bronchopulmonary aspergillosis. Chest 1985; 87: 499-502

${ }^{8}$ Patterson R, Greenberger PA, Radin RC et al. Allergic bronchopulmonary aspergillosis: staging as an aid to management. Am Int Med 1982; 96: 286-291

${ }^{9}$ Crameri R, Hemmann S, Ismail C et al. Disease-specific recombinant allergens for the diagnosis of allergic bronchopulmonary aspergillosis. Int Immunol 1998; 10: 1211 - 1216

${ }^{10}$ Greenberger PA, Miller TP, Roberts M et al. Allergic bronchopulmonary aspergillosis in patients with and without evidence of bronchiectasis. Annals of Allergy 1993; 70: 333-338

${ }^{11}$ Mendelson EB, Fisher MR, Mintzer RA et al. Roentgenographic and clinical staging of allergic bronchopulmonary aspergillosis. Chest 1985; 87: $334-339$

12 McCarthy DS, Pepys J. Allergic broncho-pulmonary aspergillosis: clinical features. Clinical allergy 1971; 1: 261-286

13 Diedrich S, Wormanns D, Heindl W. Bronchial-Carcinom-Screening mit Niedrigdosis-CT. Radiologe 2001; 41: 256-260

${ }^{14}$ Heindl W, Ernst S, Kugel H et al. Diagnostik entzündlicher Lungenerkrankungen: Vergleich von MR Bildgebung mit einer ultrakurzen Turbo-Spin-Echo Sequenz und Spiral-CT. Fortschr Röntgenstr 1997; 166: 12

${ }^{15}$ Leutner C, Schild H. MRT des Lungenparenchym. Rofo Fortschr Geb Rontgenstr N 2001; 173: 168-175

${ }^{16}$ Heußel CP, Sandner A, Voigtländer T et al. Prospektive Machbarkeitsstudie zum Vergleich von Röntgenübersichtsaufnahme und ThoraxMRT in Atemanhaltetechnik am offenen Niederfeldgerät. Fortschr Röntgenstr 2002; 174: 854-861

${ }^{17}$ Bittner RC, Felix R. Magnetic resonance (MR) imaging of the chest: state-of-the-art. Eur Respir J 1998; 11: 1392 - 1404 